\title{
Effects of osteopontin downregulation on the growth of prostate cancer PC-3 cells
}

\author{
JIE ZHENG, ZHI-BO HOU and NAN-LIN JIAO \\ Department of Pathology, School of Medicine, Southeast University, Nanjing, Jiangsu 210009, P.R. China
}

Received April 14, 2011; Accepted August 1, 2011

DOI: $10.3892 / \mathrm{mmr} .2011 .549$

\begin{abstract}
Osteopontin (OPN) has been recognized as a significant cytokine in the processes of tumorigenicity, tumor progression and metastasis in many types of human cancer. However, the functions of OPN in prostate cancer remain poorly understood. To investigate the function of OPN in human prostate cancer, the growth of prostate cancer PC-3 cells was examined following OPN downregulation by RNA interference (RNAi). PC-3 cells were transfected by two constructs containing short interfering RNAs designed to cleave two different regions of OPN mRNA. The expression of OPN in the transfected cells was markedly inhibited by RNAi at the mRNA and protein levels. Cell growth was retarded and S-phase arrest and apoptosis were observed in the transfected cells. The number and size of the colonies of the transfected cells in soft agarose were markedly decreased, as compared with those of the control cells. From these results, we conclude that the selective downregulation of OPN expression by RNAi may lead to S-phase arrest, apoptosis and a decline in the malignant phenotype in PC-3 cells, suggesting that OPN plays a significant role in the growth of prostate cancer and may be a potential therapeutic target.
\end{abstract}

\section{Introduction}

Osteopontin (OPN) is a phosphorylated glycoprotein, originally isolated from the bone extracellular matrix. OPN is expressed at constitutively low levels in a number of normal tissues, including bone, kidney, placenta, smooth muscle and secretory epithelia, and is present in various cell types, including osteoclasts, macrophages, activated $\mathrm{T}$ cells, smooth muscle cells and epithelial cells. OPN contains the cell attachment amino acid sequence RGD (arginine-glycine-aspartic acid), a binding motif that ligates cell-surface integrins. OPN has also been reported to be a

Correspondence to: Dr Jie Zheng, Department of Pathology, School of Medicine Southeast University, 87 Dingjiaqiao Road, Nanjing, Jiangsu 210009, P. R. China

E-mail: jiezheng54@126.com

Key words: osteopontin, RNA interference, prostate cancer PC-3 cells, malignant phenotype ligand of the CD44 receptor, and OPN-CD44 interactions appear to be RGD-independent. Accumulating evidence suggests that increased expression of OPN is frequently associated with tumor progression, invasion and metastasis (1-3).

Prostate cancer is one of the most common types of cancer in males, ranking as the second most common cause of cancer-related mortality in males older than 50 years of age, following carcinoma of the lung in certain Western countries. Following an initial response to androgen ablation therapy, prostate cancer invariably progresses to be androgen-independent and finally metastasizes to the bone, which is the major cause of mortality. The molecular mechanisms underlying tumor metastasis are not fully understood, but recent evidence suggests that OPN plays a significant role in the invasion, progression and metastasis of prostate cancer (4-8).

RNA interference (RNAi) is a genetic interference phenomenon induced by double-stranded RNA (dsRNA). It can specifically and efficiently degrade mRNA, resulting in posttranscriptional gene silencing. RNAi is mediated by small interfering RNAs (siRNAs), which are produced from long dsRNAs of exogenous, or more often endogenous, origin by an endonuclease of the ribonuclease-III type, known as Dicer. The resulting siRNAs are 20-22 nucleotides (nt) long and are then incorporated into the RNA-inducing silencing complex, which then recognizes and degrades mRNA containing a sequence identical to that of the siRNA.

In the present study, we used a plasmid to deliver a specially designed siRNA-targeting OPN into prostate cancer PC-3 cells, to determine whether OPN downregulation results in a declining malignant phenotype of PC-3 cells. The PC-3 cell line is an androgen-independent and tumorigenic cell line with high expression of OPN (4). The present study shows that the specific downregulation of OPN expression by RNAi is sufficient to inhibit growth, lead to S-phase arrest and apoptosis in PC-3 cells, and reduce the capacity of colony formation of PC-3 cells in soft agarose.

\section{Materials and methods}

Cell culture. The human prostate cancer cell line, PC-3, was obtained from Professor Wei-Gang Fang (Department of Pathology, Peking University, Beijing, China) and was cultured in RPMI-1640 (Gibco BRL, Grand Island, NY, USA) supplemented with $10 \%$ fetal bovine serum (FBS) (Gibco BRL) and antibiotics in a $5 \% \mathrm{CO}_{2}$ incubator at $37^{\circ} \mathrm{C}$. 
Targeting sequence 1

Predicted transcript against OPN
Targeting sequence 2

Predicted transcript against OPN

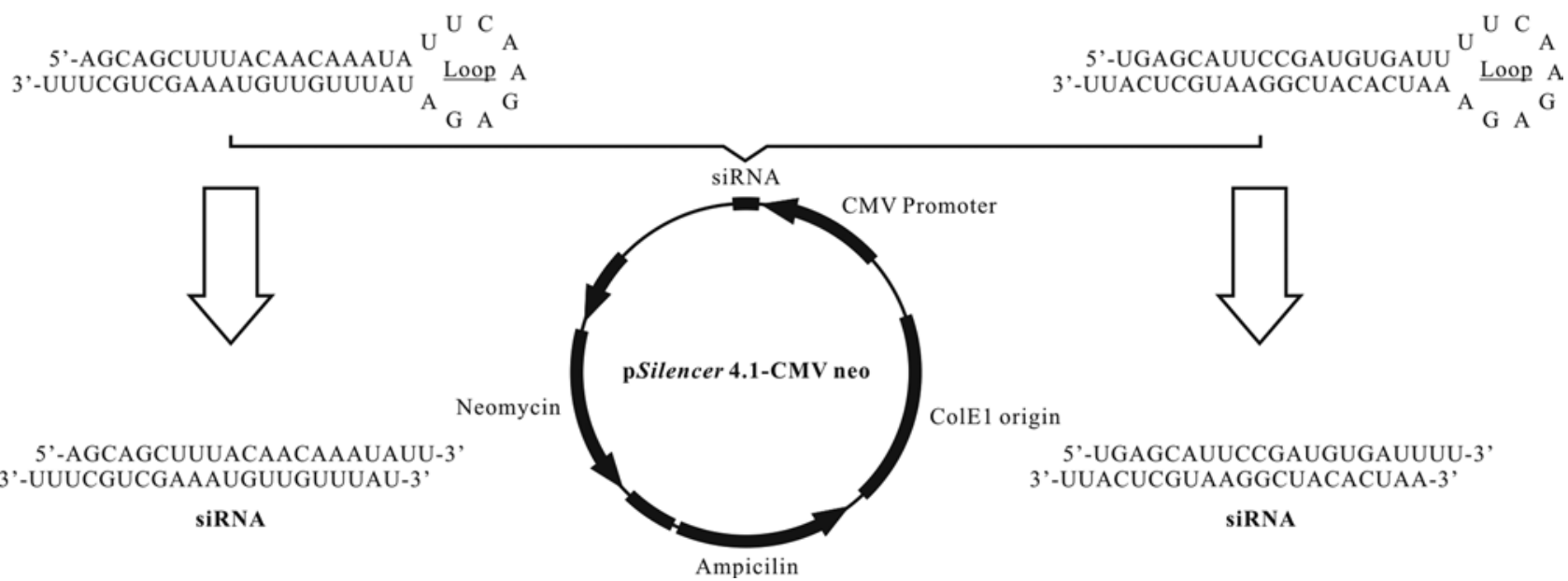

Figure 1. Molecular structure of the recombinant plasmid, pSilencer 4.1-CMV neo, and the predicted secondary structure of the transcript target, OPN. Two sequences encoding siRNA with 19 nucleotides (nt) of homology to the OPN gene were synthesized as 55 bp double-stranded DNA oligonucleotides and inserted immediately downstream of the CMV promoter. Subsequently, the hairpin loop dsRNA was transcripted and transferred into 21 nt functional siRNA by cellular enzymes that bind and destroy OPN mRNA.

Plasmid construction. The pSilencer 4.1-CMV neo (Ambion, Austin, TX, USA) vector, linearized with BamH1 and HindIII to facilitate directional cloning, was used for the construction to generate a OPN knockdown vector. The vector employs a modified cytomegalovirus (CMV) promoter to drive siRNA expression with RNA polII (Fig. 1). The two target sequences of OPN cDNA (NCBI GenBank accession no. NM000582) were designed according to the design methods of Elbashir et al and Reynolds et al siRNA $(9,10)$. These 19 -nt sequences showed no homology with other known human genes using BLAST. The hairpin siRNA sequences that contain sense and antisense sequences against OPN target 1 (pos. 252-272) and 2 (pos. 886-906) are shown in Fig. 1. Designed oligonucleotides encoding short hairpin transcripts were chemically synthesized and PAGE-purified (Invitrogen, Shanghai, China). Briefly, the short-hairpin-RNA-encoding complementary single-stranded oligonucleotides hybridized to give overhangs compatible with Bam $\mathrm{H} 1$ and HindIII were ligated into vectors. The inserts were confirmed to be in the correct sequence and orientation for expression in the new constructs by automated sequencing. The resulting plasmids were named as pSilencer-OPN S1 and pSilencer-OPN S2, respectively. The circular pSilencer 4.1-CMV neo vector that expresses a hairpin siRNA with limited homology to any known sequences in human genes was used as the negative control, named pSilencer-C. Plasmids were amplified and purified using the QIAfilter ${ }^{\mathrm{TM}}$ Plasmid Maxi kit (Qiagen, Hilden, Germany).

Transfection and selection. A total of $5 \times 10^{5}$ cells were seeded into each well of a six-well tissue culture plate (Costar). The following day, when the cells were $70-80 \%$ confluent, the culture medium was aspirated and the cells were washed with prewarmed sterile phosphate-buffered saline (PBS). The cells were transfected with the appropriate plasmids by using Lipofectamine ${ }^{\mathrm{TM}} 2000$ (Invitrogen, Carlsbad, CA,
USA) according to the manufacturer's instructions. A total of $2 \mu \mathrm{g}$ of purified pSilencer-OPN S1, pSilencer-OPN S2 and pSilencer-C were transfected into PC-3 cells and the medium was replaced with RPMI-1640 with 10\% FBS after $4 \mathrm{~h}$. Forty-eight hours after the transfections, stable transfectants were selected in $100 \mu \mathrm{g} / \mathrm{ml} \mathrm{G} 418$ in a regular medium for approximately 10 days. At this point, all of the cells in the mock plate died, and the drug resistant colonies were pooled into 6-well tissue culture plates and the cells were maintained in the presence of $100 \mu \mathrm{g} / \mathrm{ml} \mathrm{G} 418$. The stably transfected cells were named PC3OPNS1, PC3OPNS2 and PC3C, respectively.

RT-PCR analysis. RT-PCR was based on the method described by Song et al (11), with minor modifications. Briefly, total RNA was isolated with TRIzol reagent (Invitrogen) according to the manufacturer's instructions. Amplification was performed in a final volume of $25 \mu \mathrm{l}$ containing $1 \mathrm{X}$ PCR buffer, $1.5 \mu \mathrm{M} \mathrm{MgCl}_{2}, 200 \mu \mathrm{M}$ dNTP, $0.5 \mathrm{U}$ Taq DNA polymerase, $2 \mu \mathrm{l}$ template cDNA and $100 \mathrm{pM}$ of each primer. Each PCR program involved an initial denaturation step at $94^{\circ} \mathrm{C}$ for $5 \mathrm{~min}$, followed by 30 cycles (for OPN), or 28 cycles (for $\beta$-actin) at $94^{\circ} \mathrm{C}$ for $45 \mathrm{sec}, 55^{\circ} \mathrm{C}$ for $1 \mathrm{~min}$ and $72^{\circ} \mathrm{C}$ for $1 \mathrm{~min}$, and final extension at $72^{\circ} \mathrm{C}$ for $10 \mathrm{~min}$, on a PCR Thermal Cycler (Eppendorf, Hamburg, Germany). The primers used for OPN were as follows: Forward, 5'-CCCTTCCAA GTAAGTCCAACGAAAGCC-3' and reverse, 5'-GCTGAC TCGTTTCATAACTGTCCTTCCC-3' (1). The primers used for $\beta$-actin were as follows: Forward, 5'-CGTCTGGAC CTGGCTGGCCGGGACC-3' and reverse, 5'-CTAGAAGCA TTTGCGGTGGACGATG-3' (12). All primers were chemically synthesized by Invitrogen (Shanghai, China).

PCR products were resolved in a $1.7 \%$ agarose gel and stained with ethidium bromide. Gel images were captured and the band intensity was measured with Gel-Pro Analyzer 3.1 software (Media Cybernetics Inc., Silver Spring, MD, USA). The relative gene expression level was calculated as the 


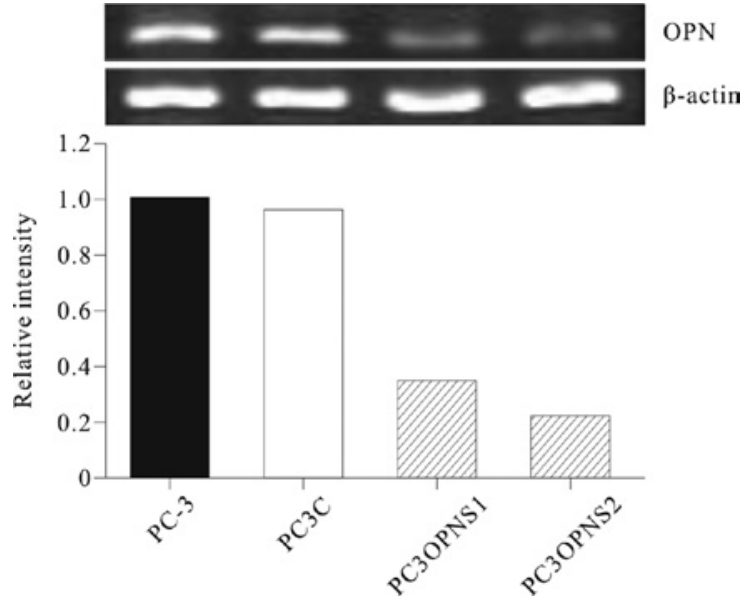

Figure 2. RT-PCR analysis showed that OPN expression in PC-3 cells was inhibited by RNAi. The illustration represents relative levels of OPN mRNA normalized with $\beta$-actin. The level of OPN mRNA was inhibited by 64 and $78 \%$ in stably transfected PC3OPNS1 and PC3OPNS2 cells, respectively, compared to the parent cells.

ratio of OPN $/ \beta$-actin, representing the OPN expression level normalized by the housekeeping gene.

Western blot analysis. For protein extracts, confluent cells were washed with cold PBS twice, denatured in lysis buffer (20 mM Tris- $\mathrm{HCl}, 200 \mathrm{mM} \mathrm{NaCl}, 0.2 \%$ Nonidet P-40 (NP-40), $0.5 \%$ Triton $\mathrm{X}-100$ and protease inhibitors) and boiled at $100^{\circ} \mathrm{C}$ for $10 \mathrm{~min}$. Equal amounts of protein extracts were separated on $10 \%$ polyacrylamide gels by using standard sodium dodecyl sulfate-polyacrylamide gel electrophoresis (SDS-PAGE) techniques, then transferred onto PVDF membranes (Bio-Rad, Hercules, California, USA). Following blocking in Tris-buffered saline (TBS) with 0.1\% Triton X-100 and 5\% milk, the membranes were incubated with mouse anti-human OPN monoclonal antibody (dilution 1:1000; Santa Cruz Biotechnology, Santa Cruz, CA, USA) and antitubulin (1:2500; Sigma, St. Louis, MO, USA) at $4^{\circ} \mathrm{C}$ overnight. Following washing, the membranes were incubated with secondary antibody goat anti-mouse conjugated to horseradish peroxidase $(1: 1000$; Sigma) at room temperature for $1 \mathrm{~h}$. Signal detection was carried out by an enhanced chemiluminescence (ECL) system (Amersham-Pharmacia Biotech). For quantification, the bands were scanned into the Gel EDAS analysis system (Cold Spring USA Corporation, Cherry Hill, NJ, USA) and analyzed by Gel-Pro Analyzer 3.1 software (Media Cybernetics, Silver Spring, MD, USA).

MTT assay. The MTT assay was used as described previously with slight modifications (12). Briefly, PC-3, PC3C, PC3OPNS1 and PC3OPNS2 cells were seeded in 96-well plates at a density of $6 \times 10^{3}$ cells per well and each group consisted of 6 parallel wells. Following incubation at $37^{\circ} \mathrm{C}$ for 24,48 and $72 \mathrm{~h}$, cell viability was determined by MTT assay.

Flow cytometric analysis. To better understand the effect of OPN downregulation on the cell cycle of transfected cells, cells were harvested and washed with ice-cold PBS twice, fixed in ice-cold $70 \%$ ethanol and stored at $-20^{\circ} \mathrm{C}$. Prior to analysis, cells were washed with PBS again, suspended in

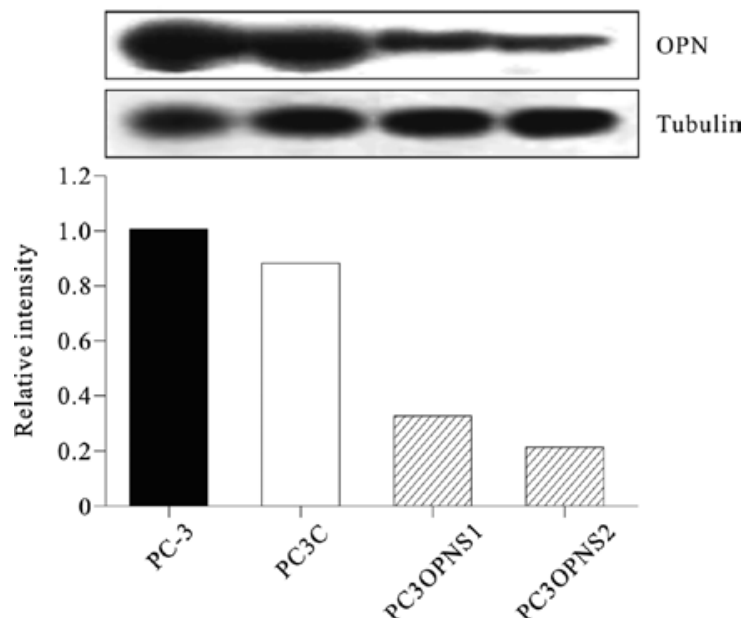

Figure 3. Western blot analysis showed that the expression of the OPN protein in PC-3 cells was inhibited by RNAi. The relative levels of OPN protein were normalized with tubulin. The level of OPN protein was inhibited by 70 and $80 \%$ in PC3OPNS1 and PC3OPNS2 cells, respectively, compared to the parent cells.

$1 \mathrm{ml}$ of cold propidium iodide (PI) solution $[0.5 \mathrm{mg} / \mathrm{ml}$ RNase, $50 \mu \mathrm{g} / \mathrm{ml} \mathrm{PI}, 0.1 \%$ (w/v) sodium citrate, and $0.1 \%$ (v/v) NP-40], and incubated on ice for $30 \mathrm{~min}$ in the dark. The DNA content of the cells was analyzed using a FACScalibur (BectonDickinson, San Jose, CA, USA) flow cytometer and ModFit LT software.

Previous studies have shown that Se-methylselenocysteine (MSC) leads to S-phase arrest and apoptosis in mouse mammary tumor TM6 cells through OPN inhibition (13-14). In order to compare the PC-3 cells by RNAi, PC- 3 cells were treated with $50 \mu \mathrm{M}$ MSC (Sigma) for $48 \mathrm{~h}$ for flow cytometric analysis. Morphology of the cells was also carefully observed.

Soft agarose growth assay. The assay for anchorage-independent growth was based on the method described by Zheng et al with minor modifications (15). In brief, PC-3, PC3C, PC3OPNS1 and PC3OPNS2 cells $\left(8 \times 10^{3}\right.$ cells per well) were mixed with complete culture medium containing agarose to result in a final agarose concentration of 0.35 and $10 \%$ FBS. Then $1 \mathrm{ml}$ samples of this cell suspension were immediately plated in 24-well plates coated with $0.6 \%$ solidified agarose on the bottom. For PC3C, PC3OPNS1 and PC3OPNS2 cells, the top and bottom culture medium included $100 \mu \mathrm{g} / \mathrm{ml} \mathrm{G} 418$. The plates were cultured in a $5 \% \mathrm{CO}_{2}$ incubator at $37^{\circ} \mathrm{C}$ for 2 weeks. The number of large colonies $(>100 \mu \mathrm{m})$ was counted in two separate experiments under a phase contrast inverted microscope (Olympus, Tokyo, Japan).

Statistical analysis. Differences between groups were determined using the Student's t-test. A value of $\mathrm{P}<0.05$ was considered to be statistically significant.

\section{Results}

Establishment of stable transfectants that silence OPN expression in PC-3 cells. To clearly understand the mechanism of OPN in prostate cancer cells, we employed RNAi to knockdown OPN expression in PC-3 cells. Using RT-PCR, we found that the levels of OPN mRNA were reduced by 64 

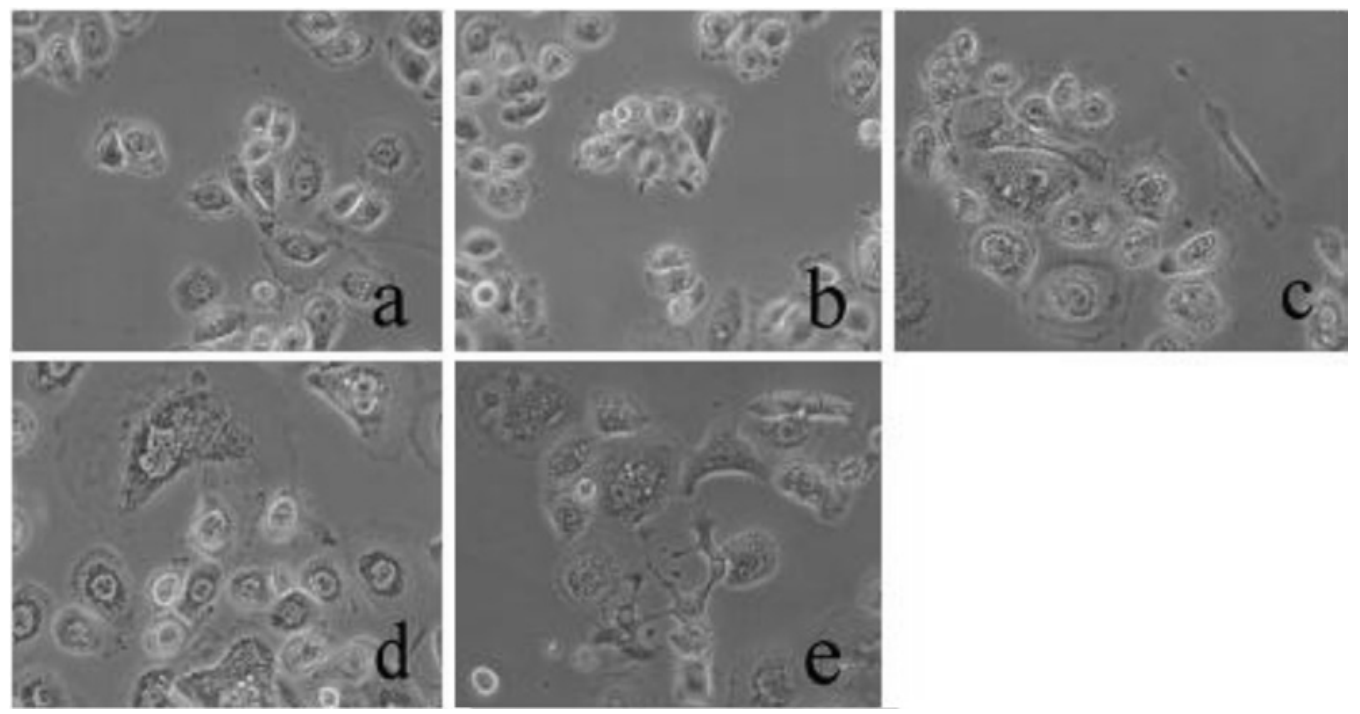

Figure 4. Morphological changes after OPN inhibition in PC-3 cells by RNAi. (a) PC-3 cells, (b) PC3C cells, (c) PC3OPNS1 cells, (d) PC3OPNS2 cells, (e) PC-3 cells treated with $50 \mu \mathrm{M}$ MSC for 2 days.

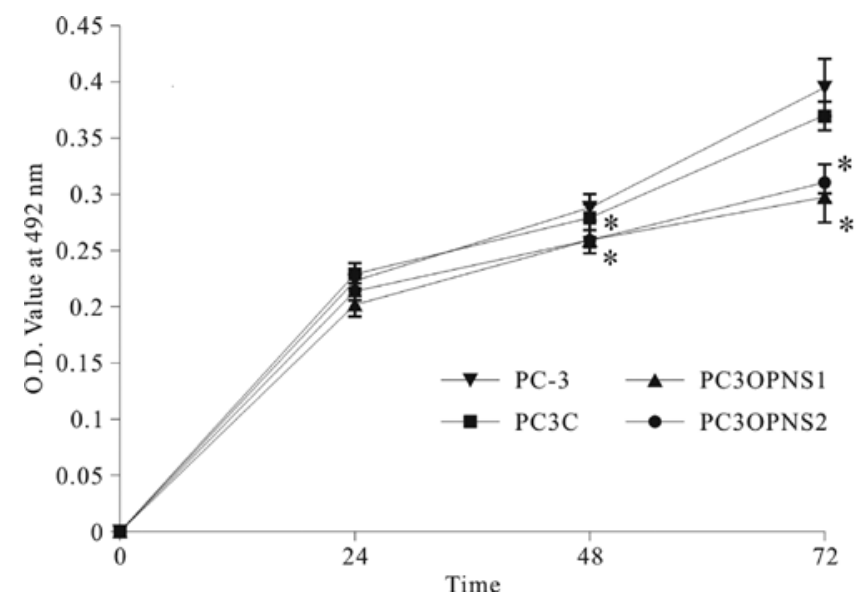

Figure 5. PC-3, PC3C, PC3OPNS1 and PC3OPNS2 cell survival was measured by MTT assay. PC3OPNS1 and PC3OPNS2 cells showed a reduced growth rate in comparison to PC-3 cells at the 48 and $72 \mathrm{~h}$ time-point after seeding. Each curve represents the mean values $( \pm \mathrm{SD})$ which were obtained from 6 wells of one group. The experiment was repeated three times with similar results. ${ }^{*} \mathrm{P}<0.05$.

and $78 \%$ in PC3OPNS1 and PC3OPNS2 cells, respectively, as compared with PC-3 cells, while the OPN expression was comparable between PC-3 and PC3C cells (Fig. 2). To confirm the RT-PCR results, Western blot analysis was carried out to determine the expression of OPN protein. Using tubulin as the internal control, endogenous OPN protein $(60 \mathrm{kDa})$ was decreased by 70 and $80 \%$ in PC3OPNS1 and PC3OPNS2 cells, respectively, compared with PC-3 cells (Fig. 3), which is consistent with the data from the RT-PCR analysis. These results clearly indicate that in our experiment, OPN mRNA in PC-3 cells was cleaved and degraded by RNAi, leading to the decrease of the corresponding protein.

Cell morphology. PC-3 cells showed epithelial-like morphology with numerous pseudopodial extensions and cell-cell contacts, and exhibited active proliferation potential.
Following transfection, the cellular morphology of PC $3 \mathrm{C}$ cells did not differ much from PC-3 cells. However, PC3OPNS1 and PC3OPNS2 cells showed significant morphological differences compared to PC-3 cells. Some of the PC3OPNS1 and PC3OPNS2 cells had a large cytoplasm with vacuoles, parts of which cracked and floated upon the cell culture fluid (Fig. 4). More vacuoles were observed in the nucleus of PC3OPNS1 and PC3OPNS2 cells in contrast to PC-3 and PC $3 \mathrm{C}$ cells. The morphological changes of PC-3 cells treated with MSC were compared with those of PC3OPNS1 and PC3OPNS2 cells.

OPN downregulation in PC-3 cells represses cell viability. The MTT assay showed that the cell viability of PC3OPNS1 and PC3OPNS2 cells was lower than the PC- 3 cells at the 48 and $72 \mathrm{~h}$ time-points (Fig. 5), and that the cell inhibition was $10.70 \%$ and $18.70 \%$ in PC3OPNS1 cells, and $10.70 \%$ and $15.70 \%$ in PC3OPNS2 cells, respectively. A statistically significant difference was observed at 48 and $72 \mathrm{~h}(\mathrm{P}<0.05)$.

Effects of OPN downregulation on cell cycle. Flow cytometer analysis (Fig. 6) showed that OPN downregulation caused a marked prolongation of the S-phase in PC3OPNS1 (16.21\%) and PC3OPNS2 (27.85\%) cells, compared with the PC-3 cells. No marked S-phase change was observed in the PC3C cells, compared with the PC-3 cells. There was a significant 'pre-G1' peak (considered as apoptotic cells) and the apoptotic index was as high as 8.27 and 7.32\% in PC3OPNS1 and PC3OPNS2 cells, respectively, compared with the PC-3 cells. By contrast, no apoptotic cells were observed in PC-3 and PC3C cells in three independent experiments. PC-3 cells treated with $50 \mu \mathrm{M}$ MSC for $48 \mathrm{~h}$ also led to S-phase arrest, which increased the percentage of cells in the S-phase by $18.97 \%$ and apoptosis by $4.55 \%$ compared with PC-3 cells (Fig. 6), which is similar to cell-cycle distributions in PC3OPNS1 and PC3OPNS2 cells.

OPN downregulation inhibits anchorage independent growth of the transfected cells. As shown in Fig. 7, OPN downregulation decreased the capacity of colony formation in PC-3 cells. 


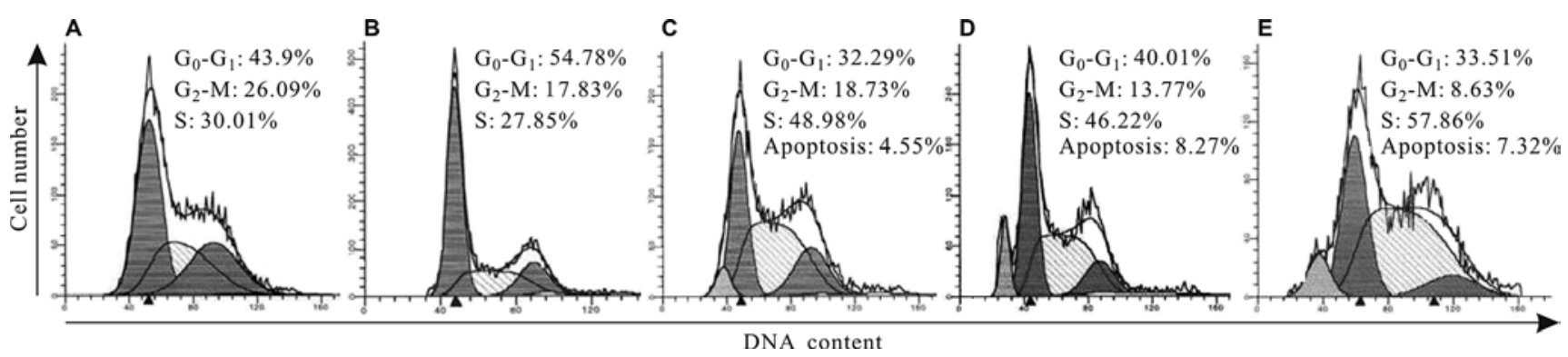

Figure 6. Flow cytometry showed that OPN knockdown induced S-phase arrest and apoptosis in PC-3 cells. (A) PC-3 cells, (B) PC 3 C cells, (C) PC-3 cells treated with $50 \mu \mathrm{M}$ MSC, (D) PC3OPNS1 cells and (E) PC3OPNS2 cells. The experiment was repeated twice or more with similar results.

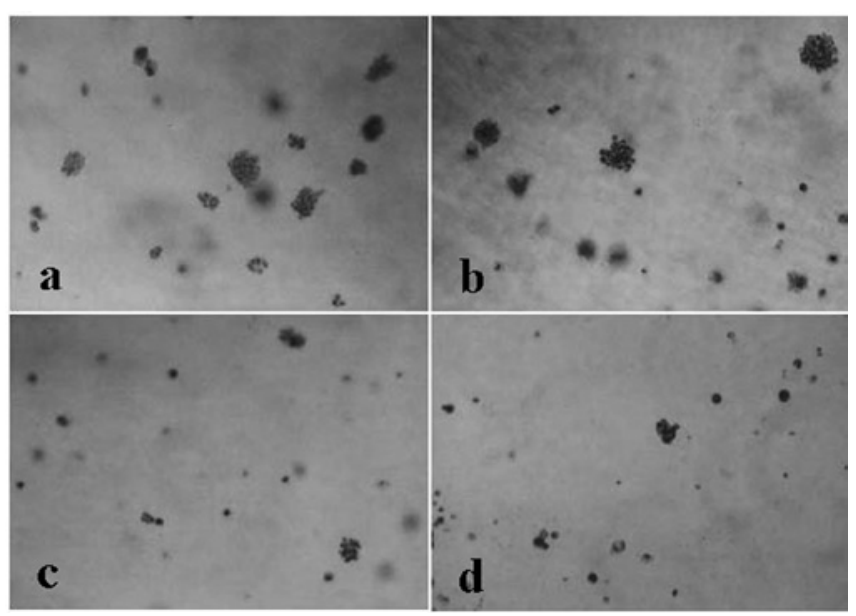

Figure 7. OPN downregulation decreased the capacity of colony formation of cancer cells in a soft agarose growth assay (magnification, $x 40$ ). (a) PC-3 cells, (b) PC3C cells, (c) PC3OPNS1 cells and (d) PC3OPNS2 cells.

In two independent experiments, the PC-3 and PC3C cells formed more colonies (average of 52 and 47, respectively) than the PC3OPNS1 and PC3OPNS2 cells (average of 14 and 9, respectively) following 14-day culture $(\mathrm{P}<0.05)$.

\section{Discussion}

OPN is one of the matricellular proteins and has been shown to be involved in the cell proliferation, migration and metastasis of tumor cells. Experimental studies have found that clinical prostate tumor specimens of high Gleason grade or metastatic specimens express high levels of OPN and markedly support the idea that increased OPN expression correlates with human prostate cancer progression and metastasis (4). In models of breast cancer and melanoma, OPN has been shown to activate molecular mechanisms that regulate the progression and malignant behavior of tumor cells $(2,16)$. However, there are currently few studies that describe the precise role of OPN in models of human prostate cancer. Therefore, we examined the effects of OPN downregulation on the growth of PC-3 cells.

RNAi is a powerful technique for the analysis of gene function in eukaryotes and makes gene inactivation possible in organisms that were not previously amenable to analysis of gene function. The RNAi-directed effective gene knockdown depends on the appropriate selection of the target mRNA sequence. First, we selected the candidate siRNA sequence according to empirical criteria for siRNA design established by Elbashir et al (9). However, not every sequence based on the criteria is effective. To achieve a more effective siRNA sequence, we selected two target sequences in the candidate siRNA referenced from a previous report for rational siRNA selection (10). The characteristics associated with siRNA function were identified: Low G/C content, a bias towards low internal stability at the sense strand 3'-terminus, lack of inverted repeats and sense strand base preferences (positions 3, 11, 14 and 20). The present study shows that transfections of the two siRNA expression vectors designed to target OPN, specifically and effectively inhibited OPN expression by 64 and $70 \%$ in PC3OPNS1 cells and 78 and $80 \%$ in PC3OPNS2 cells at the mRNA and protein levels, respectively, whereas the OPN expression of the PC3C cells was comparable to that of the PC-3 cells at the mRNA and protein levels, suggesting that our designed siRNA sequences were specific and effective. Previous reports have shown that transfection with antisense or siRNA against OPN may lead to reduced malignant and metastatic potentials $(8,17-20)$. However, there are few reports that elucidate the possible role that OPN plays in prostate cancer (4). To the best of our knowledge, this is the first report of OPN expression being downregulated by RNAi in human prostate cancer PC-3 cells. The present study provides a model to explore the potential mechanism by which OPN controls prostate cancer progression and the malignant phenotype.

In morphology, PC3OPNS1 and PC3OPNS2 cells are quite different from the parental cells. Some of the PC3OPNS1 and PC3OPNS2 cells had a large cytoplasm with vacuoles, parts of which were cracked and floated upon the cell culture fluid, consistent with a previous study that showed that the inhibition of OPN expression by antisense oligonucleotides led to radiated morphological changes in human oral cancer BSC-OF cells (18). The morphological changes mentioned above indicate that OPN downregulation may alter the cytoplasmic skeleton pattern via its receptors, e.g. CD44 and integrins, in an autocrine manner (21).

To date, there is little evidence showing that OPN is related to cell cycle and apoptosis in cancer cells. MSC is considered an attractive selenium compound for cancer chemoprevention based on its organic nature, low toxicity and substantial anticarcinogenic properties (22). Sinha and Medina demonstrated that MSC led to cell cycle arrest in TM6 mouse mammary tumor cells, which was followed by the cells entering apoptosis (13). A later study using cDNA array analysis indicated 
that OPN is a potential target gene in the inhibition of mammary tumorigenesis by MSC, and this was subsequently confirmed by RT-PCR (14). Therefore, PC-3 cells were treated with MSC to see whether we could achieve identical results using RNAi. MSC (50 $\mu \mathrm{M})$ led to S-phase arrest and apoptosis in PC-3 cells, which was similar to the S-phase arrest and apoptosis induced by OPN downregulation using RNAi.

OPN has been shown to prevent apoptosis and confer an IL-3-mediated survival advantage on murine B cells through activation of the phosphatidylinositol 3-kinase/protein kinase B (PI3K/Akt) pathway (23). However, little evidence on OPN-induced apoptosis in cancer cells has been reported up to now. In this study, we demonstrate that OPN downregulation induces apoptosis in PC-3 cells, thus suggesting that OPN plays a critical role in apoptosis in prostate cancer. Notably, another study has suggested that genistein may delay the progression from benign to malignant tumors by inhibiting OPN expression in prostatic adenocarcinoma (24). These findings have provided sufficient evidence that OPN indeed plays a role in the progression of prostate cancer and may be a potential therapeutic target in prostate cancer.

OPN stimulates androgen-dependent prostate cancer LNCaP cell proliferation in serum-free medium and colony growth at a high dilution (5). OPN secreted by macrophages is capable of restoring the metastatic potential of OPN-knockdown cancer cells (25), indicating that cancer cells may take advantage of growth by OPN in an autocrine or paracrine manner. A previous study has revealed an elevated expression of OPN mRNA in the androgen-independent and tumorigenic prostate cancer cell lines, C4-2, DU-145, and $\mathrm{PC}-3$, and to a lesser extent in the androgen-dependent LNCaP cell line (4). Our results support the notion that the progression towards increased malignant potential of androgen-dependent prostate cancer cells is possibly sustained during clonal selection by high concentrations of paracrine growth factors, and this mechanism may be amplified by a synergistic interaction between epidermal growth factor (EGF) and OPN (26). The malignant phenotype is determined by anchorage-independent growth in semisolid media such as agar or agarose, which is highly consistent with tumor formation in nude mice. The capacity of colony formation by PC3OPNS1 and PC3OPNS2 cells was markedly reduced compared with PC-3 and PC3C cells, consistent with previous studies that show that OPN contributes to prostate cancer cell anchorage-independent growth in soft agar (4), suggesting the possibility that OPN overproduction is causally related to transformation of preneoplastic cells.

In conclusion, our results show that OPN depletion results in growth inhibition, S-phase arrest, apoptosis of PC-3 cells and the decreased malignant phenotype of PC-3 cells. We conclude that OPN may confer selective growth, progression and malignant potential to human prostate cancer cells. This noteworthy result may have significant clinical implications and OPN may be a potential target for prostate cancer treatment.

\section{Acknowledgements}

This study was in part supported by a grant from the Ministry of Education, China (no. 6823001004). We thank
Dr Wei-gang Fang for generously providing the PC-3 cells. We would like to thank Xue-feng Fang, Ming-kuan Sun and Cong Chen for their suggestions and technical assistance. Dr Peng Gao is acknowledged for art work of the figures.

\section{References}

1. Pan HW, Ou YH, Peng SY, Liu SH, Lai PL, Lee PH, Sheu JC, Chen CL and Hsu HC: Overexpression of osteopontin is associated with intrahepatic metastasis, early recurrence, and poorer prognosis of surgically resected hepatocellular carcinoma. Cancer 98: 119-127, 2003.

2. Zhou Y, Dai DL, Martinka M, Su M, Zhang Y, Campos EI, Dorocicz I, Tang L, Huntsman D and Nelson C: Osteopontin expression correlates with melanoma invasion. $\mathrm{J}$ Invest Dermatol 124: 1044-1052, 2005.

3. Weber GF, Lett GS and Haubein NC: Osteopontin is a marker for cancer aggressiveness and patient survival. Br J Cancer 103: 861-869, 2010.

4. Thalmann GN, Sikes RA, Devoll RE, Kiefer JA, Markwalder R, Klima I, Farach-Carson CM, Studer UE and Chung LW: Osteopontin: possible role in prostate cancer progression. Clin Cancer Res 5: 2271-2277, 1999.

5. Angelucci A, Festuccia C, D'Andrea G, Teti A and Bologna M: Osteopontin modulates prostate carcinoma invasive capacity through RGD-dependent upregulation of plasminogen activators. Biol Chem 383: 229-234, 2002

6. Forootan SS, Foster CS, Aachi VR, Adamson J, Smith PH, Lin K and Ke Y: Prognostic significance of osteopontin expression in human prostate cancer. Int J Cancer 118: 2255-2261, 2006.

7. Robertson BW, Bonsal L and Chellaiah MA: Regulation of Erk1/2 activation by osteopontin in PC3 human prostate cancer cells. Mol Cancer 9: 260-270, 2010.

8. Zhang Y, Forootan SS, Kamalian L, Bao ZZ, Malki MI, Foster CS and Ke Y: Suppressing tumourigenicity of prostate cancer cells by inhibiting osteopontin expression. Int J Oncol 38: 1083-1091, 2011.

9. Elbashir SM, Harborth J, Weber K and Tuschl T: Analysis of gene function in somatic mammalian cells using small interfering RNAs. Methods 26: 199-213, 2002.

10. Reynolds A, Leake D, Boese Q, Scaringe S, Marshall WS and Khvorova A: Rational siRNA design for RNA interference. Nat Biotechnol 22: 326-330, 2004.

11. Song J, Zhang DS and Zheng J: Expression of CD44v6 gene in normal human peripheral blood. World J Gastroenterol 11: 3591-3594, 2005.

12. Zheng J, Deng YP, Lin C, Fu M, Xiao PG and Wu M: Arsenic trioxide induces apoptosis of HPV16 DNA-immortalized human cervical epithelial cells and selectively inhibits viral gene expression. Int J Cancer 82: 286-292, 1999.

13. Sinha $\mathrm{R}$ and Medina D: Inhibition of $\operatorname{cdk} 2$ kinase activity by methylselenocysteine in synchronized mouse mammary epithelial tumor cells. Carcinogenesis 18: 1541-1547, 1997.

14. Unni E, Kittrell FS, Singh U and Sinha R: Osteopontin is a potential target gene in mouse mammary cancer chemoprevention by Se-methylselenocysteine. Breast Cancer Res 6: R586-R592, 2004.

15. Zheng J and Vaheri A: Human skin fibroblasts induce anchorageindependent growth of HPV-16-DNA-immortalized cervical epithelial cells. Int J Cancer 61: 658-665, 1995.

16. Tuck AB and Chambers AF: The role of osteopontin in breast cancer: clinical and experimental studies. J Mammary Gland Biol Neoplasia 6: 419-429, 2001.

17. Behrend EI, Craig AM, Wilson SM, Denhardt DT and Chambers AF: Reduced malignancy of ras-transformed NIH 3T3 cells expressing antisense osteopontin RNA. Cancer Res 54: 832-837, 1994.

18. Muramatsu T, Shima K, Ohta K, Kizaki H, Ro Y, Kohno Y, Abiko $\mathrm{Y}$ and Shimono M: Inhibition of osteopontin expression and function in oral cancer cell lines by antisense oligonucleotides. Cancer Lett 217: 87-95, 2005.

19. Wai PY, Mi Z, Gou H, Sarraf-Yazdi S, Gao C, Wei J, Marroquin CE, Clary B and Kuo PC: Osteopontin silencing by small interfering RNA suppresses in vitro and in vivo CT26 murine colon adenocarcinoma metastasis. Carcinogenesis 26: 741-751, 2005.

20. Gong M, Lu Z, Fang G, Bi J and Xue X: A small interfering RNA targeting osteopontin as gastric cancer therapeutics. Cancer Lett 272: 148-159, 2008. 
21. Denhardt DT, Noda M, O'Regan AW, Pavlin D and Berman JS: Osteopontin as a means to cope with environmental insults: regulation of inflammation, tissue remodeling, and cell survival. J Clin Invest 107: 1055-1061, 2001.

22. Ip C, Hayes C, Budnick RM and Ganther HE: Chemical form of selenium, critical metabolites, and cancer prevention. Cancer Res 51: 595-600, 1991.

23. Lin YH and Yang-Yen HF: The osteopontin-CD44 survival signal involves activation of the phosphatidylinositol 3-kinase/ Akt signaling pathway. J Biol Chem 276: 46024-46030, 2001.

24. Mentor-Marcel R, Lamartiniere CA, Eltoum IA, Greenberg NM and Elgavish A: Dietary genistein improves survival and reduces expression of osteopontin in the prostate of transgenic mice with prostatic adenocarcinoma (TRAMP). J Nutr 135: 989-995, 2005.
25. Cheng J, Huo DH, Kuang DM, Yang J, Zheng L and Zhuang SM: Human macrophages promote the motility and invasiveness of osteopontin-knockdown tumor cells. Cancer Res 67: 5141-5147, 2007.

26. Angelucci A, Festuccia C, Gravina GL, Muzi P, Bonghi L, Vicentini $\mathrm{C}$ and Bologna M: Osteopontin enhances the cell proliferation induced by the epidermal growth factor in human prostate cancer cells. Prostate 59: 157-166, 2004. 